\title{
Research and Application of Intelligent Remote Anti-electricity-stealing Monitoring System
}

\author{
Shengli Xie ${ }^{1}$, Xiaodong Zhang ${ }^{1}$,Bo Liu1, BoNing ${ }^{2}$, Lizong Wang ${ }^{3}$ \\ 1.Skills Training Center of State Grid Electric Power Co., Ltd in Northern Hebei, Baoding, Hebei, \\ 071003, China \\ 2.Marketing Center of State Grid Electric Power Co., Ltd in Northern Hebei, Beijing, 100000,China \\ 3.Beijing Xinyuan Green Net Energy Technology Co., Ltd, Beijing, 100000,China
}

Keywords:anti-electricity-stealing; remote monitoring; fault diagnosis.

\begin{abstract}
In view of the line loss control and the anti-electricity-stealing requirements faced by electric power companies, the design of the intelligent anti-electricity-stealing remote monitoring system has been proposed. The real-time monitoring of primary side of line current, the current of junction box and the current of metering device has been employed to determine the existence of electricity-stealing behavior and the specific location of electricity-stealing outlier. At the same time, the composition of intelligent anti-electricity-stealing remote monitoring system as well as the key technologies has been introduced in this paper. The application results show that this system can quickly diagnose the electricity-stealing behaviors of users and accurately determine the specific location of electricity-stealing outliers. The effect of anti-electricity-stealing is obvious.
\end{abstract}

\section{Introduction}

For a long time, a small number of power users have taken various illegal means to steal the electricity driven by the interests. Especially for $10 \mathrm{kV}$ users, the electric power loss will be more serious in the occurrence of abnormal electrical behaviors or electricity-stealing behaviors due to the larger power consumption. With the development of technology, the electricity-stealing technology has been increasingly intelligent and industrial. The electricity-stealing behavior is more subtle. In some regions, some users have frequently installed the transfer switch and the wireless remote control switch in shelters to implement the remote electricity-stealing. The electricity-stealing methods are increasingly subtle and the technology content of electricity-stealing is also increasing. It is difficult to monitor the behaviors of current diverting, electricity-stealing of strong magnet and the modification of electric meter parameters by intelligent infrared remote control. The losses of electric quantity in power supply enterprises are heavy and these companies have suffered huge economic losses each year.

In view of the line loss control and the anti-electricity-stealing requirements faced by electric power companies, the intelligent remote electricity-stealing monitoring system based on electricity-stealing principles has been designed through the in-depth study on the electrical anomaly detection of $10 \mathrm{kV}$ users and the diagnosis models. In this paper, the design programs of system, the composition framework and the employed key technologies have been proposed so that the monitoring system can implement the 24 hours real-time monitoring for the special transformer users. At the same time, the electricity-stealing behaviors can be effectively suppressed and the normal order of electricity can be effectively maintained.

\section{Design of intelligent remote electricity-stealing monitoring system}

In the electric power system, the electricity-stealing methods of metering box are various, which can be divided into the following five types: voltage circuit stealing, current loop stealing, the wrong phase sequence electricity-stealing mode, the high-tech (remote control/ strong magnet/ high-frequency interference) electricity-stealing and the secretly lap jointing stealing[1-2]. As the measuring signals of metering box are introduced by transformer, it is vulnerable to destroy them. 
Therefore, the good anti-electricity-stealing effect can not be received through solely relying on improving the metering box and other technologies.

Therefore, in the design of remote monitoring system, it is required to employ the high-voltage detection components to collect the primary side data of users (before metering and after metering), adapt the current collection device to collect the current data (front the terminal box and behind the terminal box) and use the RS485 port to real-timely read the current data in meter. Through comparing with the three data, the specific location of abnormal points can be analyzed and positioned. The installation of intelligent remote power monitoring system is shown in Figure 1 (taking $10 \mathrm{kV}$ high-supply and high-count users as examples).

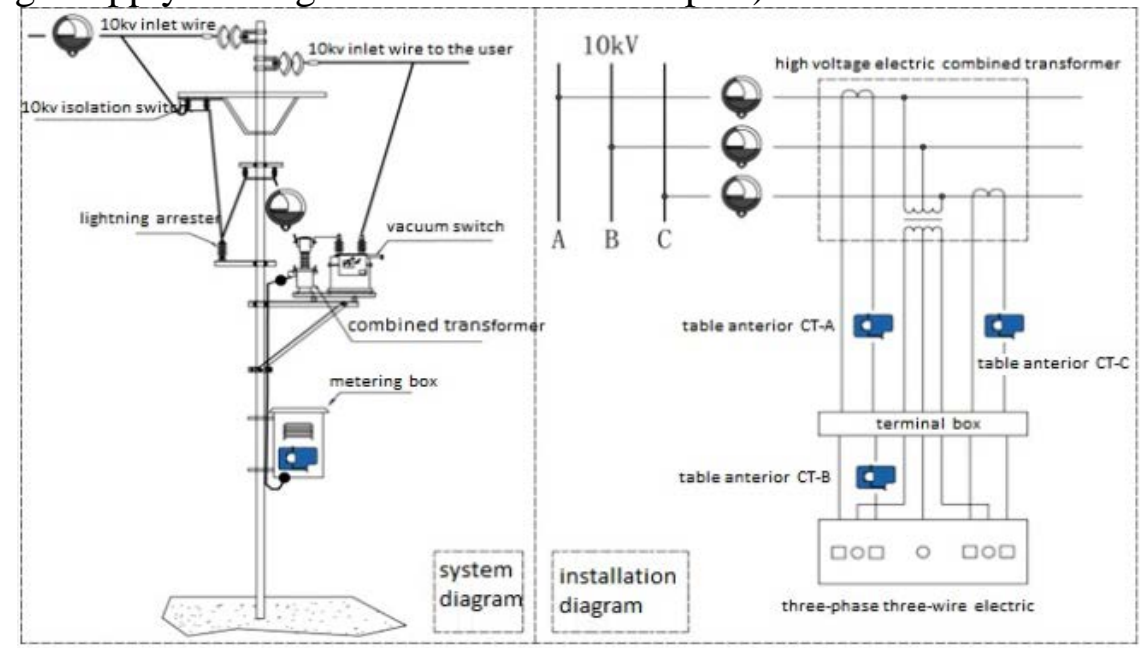

Figure.1: Installation of anti-electricity-stealing system

$10 \mathrm{kV}$ primary side line is sent to users through isolation switch, modular transformers and vacuum switches. The modular transformer sends the current signals to metering box through junction box for measuring. It is required to measure the current data of primary side line, the current data front the meter and the data in meter. If current data of primary side line in front of metering box and behind the metering box have relatively large differences, it can be determined that the users are suspected of stealing electricity. Thus, it is necessary to compare the data before the terminal box and after the terminal box. If they are not consistent, the outliers can be determined in the terminal box; if they are consistent with each other, the outliers can be determined in metering box.

\section{Composition of intelligent remote anti-electricity-stealing monitoring system}

The intelligent remote anti-electricity-stealing monitoring system is composed by anti-electricity-stealing user master system, data loggers, high-voltage wireless detection units, intelligent magnetic field detection units, measuring transformers and handheld terminals, etc, which are shown in Figure 2.

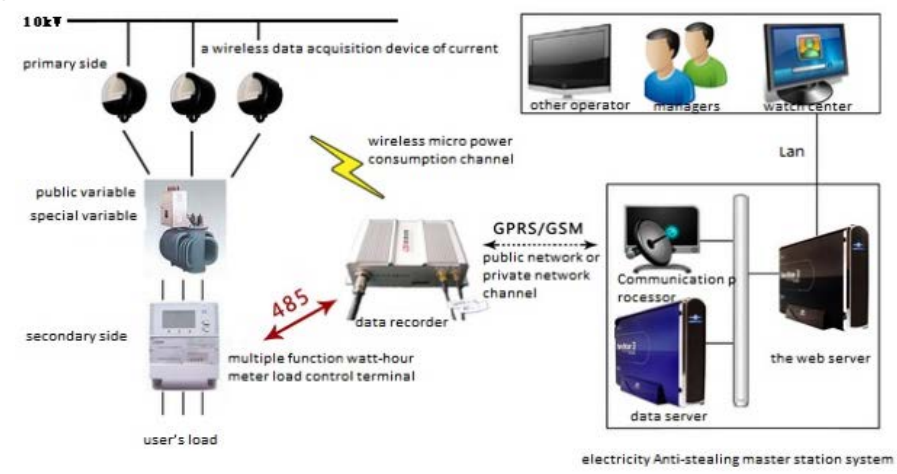

Figure.2: Intelligent remote anti-electricity-stealing monitoring system 
1) Anti-electricity-stealing user master system can realize the back-stage management of anti-electricity-stealing technology through combining the server with the smartphones. At the same time, the abnormal situations of operation of each branch line and each user can be analyzed and recorded.

The anti-electricity-stealing master system is the key part of whole system. It has a series of functions to ensure the safe and meter operation of whole system. The master system is divided into the communication master and the application master. The function of communication master is to receive the alarming information in alarm terminal and issue the various operating instructions to alarm terminals. The application master employs the web platform designed by the three-tier B/S architecture to manage the basic file information and various alarming information such as user information, alarming information and traffic card information[3].

The alarm terminal is the core part of whole system that is designed as the open multiple protective mode. It supports the following communication modes: GPRS and CDMA. The alarm has the functions of door-opening alarm, vibration alarm, strong magnetic alarm, power failure alarm, infrared alarm and wireless alarm. It can real-timely monitor the operation of metering device and the surrounding electromagnetic environment of metering device.

The data logger uses the embedded real-time multitasking operating system to analyze the obtained data. The device has two wireless communication interfaces, a RS485 communication interface and a USB maintenance interface. Through installing the ultra-low-power wireless communication module, it is required to real-timely collect the three-phase current data of high-voltage wireless detection units. Through RS485 interface, it is communicated with meter; it is necessary to upload the primary side and the secondary side whole data and send the alarming information of master.

The data logger is communicated with the secondary side meter to real-timely record and monitor the voltage loop and current loop. When the anomalies such as under voltage, loss of voltage, diversion of current, opening of circuit, phase shift, interference of strong magnetic and loss of power are appeared, the alarming information will be sent to the set phone through messages.

The data logger measures an actual load and metering load. When the deviation is greater than the given value, it is determined that the electricity load of the user is abnormal. Then, the remote monitoring device will send the alarming information to the given phone through messages[4].

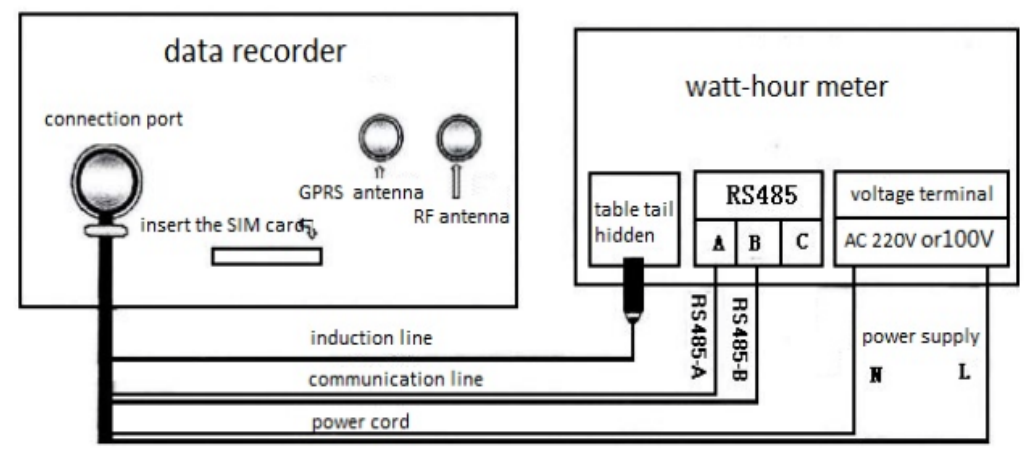

Figure.3: Connection diagram of data logger and electric energy meter

The high-voltage wireless detection unit is a passive high-voltage wireless current transformer, which is composed by Rogowski coil, power coils, wireless communication module and main module. It can be charged to be disassembled. The high-voltage wireless detection unit measures and calculates the current to obtain the current value. Through the micro-power wireless circuit, the current value is sent to the data logger or GPRS/GSM service terminals[5].

4) The intelligent magnetic detection unit is employed to realize the online monitoring function of magnetic field and electromagnetic wave.

5) The measuring transformer is used to measure the current data in front of meter and it has the positioning function.

6) The handheld terminal is used to set all parameters of data logger and view the data collected by data logger. The secondary current of circuit is measured and the changes of current transformer can be calculated by comparing with the primary current collected by high-voltage clamp meter. 


\section{Application analysis of intelligent remote anti-electricity-stealing monitoring system}

The intelligent remote anti-electricity-stealing monitoring system is based on B/S web platform. The system is composed as the active anti-electricity-stealing system with the high-voltage wireless detection unit, the data logger, the transformers and other remote monitoring terminals. This system can not only monitor whether the users have the stealing behaviors, but also identify the possible ways of high-tech electricity-stealing.

The application of intelligent remote anti-electricity-stealing monitoring system can be illustrated by taking the internal part of meter and the wireless remote electricity-stealing method as examples. In Figure 4, the adjustable resistance can randomly adjust the metering current loop through wireless remote control so as to promote the meter to calculate less electric quantity. Or the remote control switch is added into the voltage circuit. The metering voltage circuit can be opened through remote control to cause the fault of metering voltage circuit. Then, the voltage coils of electric energy meter are under voltage and the electric energy meter does not count. The stealers can easily implement the remote control according to their requirements.

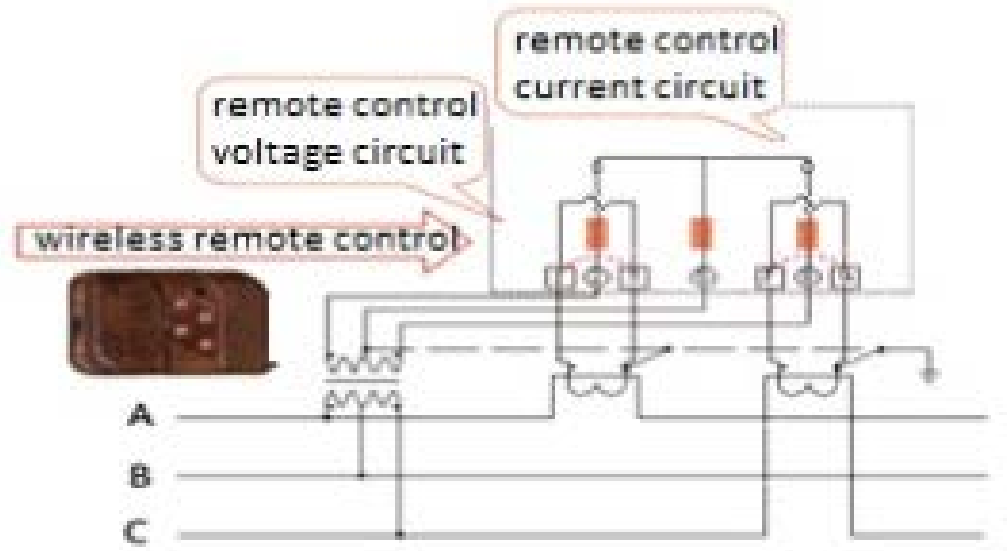

Figure.4: The addition of wireless remote control into the meter for electricity-stealing

The wireless sensors can respectively send the primary current of $10 \mathrm{kv}$ specific transformer, the current in front of meter and the current of meter to the data logger. The data will be sent to the master system by data logger and it can be judged whether these exist the stealing behavior through analysis.

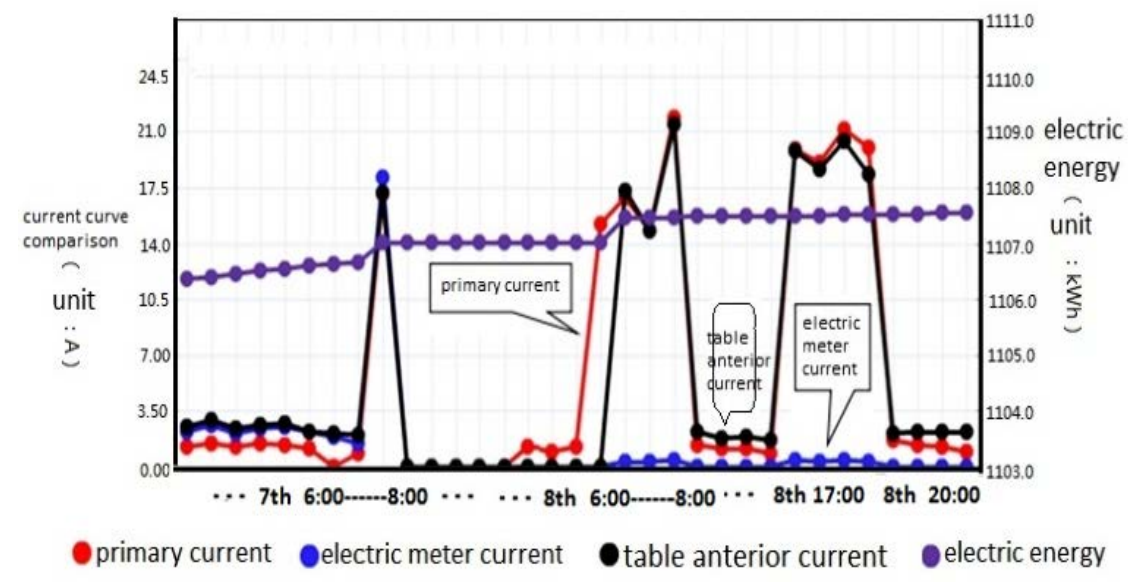

Figure.5: Contrast curve of current

In Figure 5, it is found that on the 7th day, the users begin to produce at 6:00-- 8:00; the primary current, the current in front of meter and the current of meter remain the same; on the 8th day, the current of meter is less than 97\% of primary current and current in front of meter at 15:00 --- 20:00. Therefore, it can be determined that the users have the obvious electricity-stealing behaviors. As the 
primary side current is consistent with the current in front of meter, they are far greater than the current of meter. Therefore, it can be judged that the position of abnormality is located in meter and the loss of power can be easily calculated, namely: the loss of electric quantity= primary electric quantity - electric quantity of meter.

\section{Conclusion}

In the checking process of monitoring electricity-stealing, the monitoring of user's actual load (i.e. primary electrical quantity of transformer) is the basis to quickly and accurately detect the electricity-stealing. The intelligent remote anti-electricity-stealing monitoring system is the new technology and the new means to realize this purpose.

Through the 24-hour online monitoring for suspicious users, it can be found whether the user has the electricity-stealing behaviors. At the same time, in the process of line loss management, the serious line loss circuit can be monitored line by line to form the three-dimensional monitoring network. The management level of line loss of $10 \mathrm{kv}$ network can be greatly improved through significantly reducing the line loss rate of $10 \mathrm{kv}$ public lines.

\section{References}

[1] Zhang Gang. Development of Intelligent Anti-electricity-stealing Device of Large Electricity Users[Master's Thesis]. Dalian University of Technology, 3,pp.1-5,2000.

[2] Huang Wenke. Research on Remote Intelligent Anti-electricity-stealing Management System[Master’s Thesis]. South China University of Technology, 10,pp.3-5,2006.

[3] Liu Kunpeng, Yu Haifeng, Shao Hongtao, Bi Xifei. Research and Application of Anti-electricity-stealing Real-time Alarming Management System[J]. Anhui Electrical Engineering Professional Technique College, 10, pp.14-17,2011.

[4] Su Kun. Research and Application of Anti-electricity-stealing Technology of Power Distribution Network in Baoding[Master’s Thesis]. North China Electric Power University, 3,pp.1-4,2012.

[5] Beijing Xinyuan Green Net Energy Technology Co., Ltd. Passive high-voltage Wireless Current Transformer. China, 200820180226. 4[P]. 2008, 11, 28. 